\title{
Nonlinear deconvolution with deblending: a new analyzing technique for spectroscopy
}

\author{
C. Sennhauser ${ }^{1}$, S. V. Berdyugina ${ }^{1,2}$, and D. M. Fluri ${ }^{1}$ \\ 1 Institute for Astronomy, ETH Zurich, 8093 Zurich, Switzerland \\ e-mail: csennhau@astro.phys.ethz.ch \\ 2 Kiepenheuer Institut für Sonnenphysik, 79104 Freiburg, Germany
}

Received 11 May 2009 / Accepted 1 August 2009

ABSTRACT

\begin{abstract}
Context. Spectroscopy data in general often deals with an entanglement of spectral line properties, especially in the case of blended line profiles, independently of how high the quality of the data may be. In stellar spectroscopy and spectropolarimetry, where atomic transition parameters are usually known, the use of multi-line techniques to increase the signal-to-noise ratio of observations has become common practice. These methods extract an average line profile by means of either least squares deconvolution (LSD) or principle component analysis (PCA). However, only a few methods account for the blending of line profiles, and when they do, they assume that line profiles add linearly.

Aims. We abandon the simplification of linear line-adding for Stokes $I$ and present a novel approach that accounts for the nonlinearity in blended profiles, also illuminating the process of a reasonable deconvolution of a spectrum. Only the combination of those two enables us to treat spectral line variables independently, constituting our method of nonlinear deconvolution with deblending (NDD). The improved interpretation of a common line profile achieved compensates for the additional expense in calculation time, especially when it comes to the application to (Zeeman) doppler imaging (ZDI).

Methods. By examining how absorption lines of different depths blend with each other and describing the effects of line-adding in a mathematically simple, yet physically meaningful way, we discover how it is possible to express a total line depth in terms of a (nonlinear) combination of contributing individual components. Thus, we disentangle blended line profiles and underlying parameters in a truthful manner and strongly increase the reliability of the common line patterns retrieved.

Results. By comparing different versions of LSD with our NDD technique applied to simulated atomic and molecular intensity spectra, we are able to illustrate the improvements provided by our method to the interpretation of the recovered mean line profiles. As a consequence, it is possible for the first time to retrieve an intrinsic line pattern from a molecular band, offering the opportunity to fully include them in a NDD-based ZDI. However, we also show that strong line broadening deters the existence of a unique solution for heavily blended lines such as in molecular bandheads.
\end{abstract}

Key words. line: formation - stars: magnetic fields

\section{Introduction}

A multi-line approach for increasing the signal-to-noise $(\mathrm{S} / \mathrm{N})$ ratio of measured line profiles was first introduced by Semel (1989) and Semel \& Li (1996). It was developed into the LSD method by Donati et al. (1997). The aim of these methods is to determine the common Stokes profiles of polarized spectra when assuming the weak-field regime. It is assumed that the line profiles are the same for all lines in the spectrum except for a factor, which is given by the properties of the transition $|i\rangle \rightarrow|f\rangle$, i.e., wavelength, effective Landé factor, and oscillator strength, and by the stellar atmosphere in which the lines form, i.e., shape and depth of the (intensity) line. These mean Stokes profiles are called common line patterns, or Zeeman patterns $Z(v)$ of a spectrum, which are presented in velocity space. The spectrum is then a correlation of this common line pattern with a linemask, which defines the line position (wavelength) and scaling in intensity. Another approach is that of principal component analysis (PCA), where denoising is achieved by diagonalizing a cross-product matrix of individual spectral lines to reconstruct the data with a truncated basis of eigenvectors (e.g., Martínez Gonzáles et al. 2008).
Methods such as LSD and PCA can be assumed to be filtering methods, which extract common line patterns of Stokes $I, Q, U$, and $V$ in more detail, thus enabling a more precise description of the stellar atmosphere in which the lines formed, including the temperature and both the strength and orientation of the magnetic fields. For the task of Zeeman doppler imaging (ZDI), that reconstructs the brightness and magnetic field distribution on a stellar surface from input multiple exposures at different rotational phases, such a multi-line technique seems to be an appropriate tool for providing highly resolved information about the star merged in one single line profile.

To retrieve this common line pattern, it is insufficient to represent a spectrum as an accumulation of separate spectral lines, but it is necessary to deconvolve blended line profiles, i.e., to account for the contributions of closely neighbouring lines (in all Stokes parameters). So far, LSD methods assume that contributions from different lines add up linearly for all Stokes parameters, i.e., that the spectrum is a true convolution in a mathematical sense. We show that this is only valid for weak absorption lines, and we present a self-consistent way of analyzing arbitrary line depths. The main goal was to find a formalism that describes how line depths "add" in a physical sense, which we 
found in terms of the interpolation formula by Minnaert (1935), introduced for quite a different purpose.

In this paper, we consider intensity spectra only, while a consistent procedure for polarized spectra will be developed in a forthcoming paper. The paper is structured as follows. In Sect. 2, we consider the basics of multi-line techniques, introducing the principles of deblending. We then compare optically thin and thick cases in Sect. 3, to develop a general formalism from which we derive our nonlinear deconvolution with deblending (NDD) technique. The numerical implementation of our new method is given in Sect. 4, which is supplemented by Appendices A-C, where we provide details about inverse interpolation, solving algorithms, and defining the Jacobi matrix explicitly. A comparison of linear and nonlinear deconvolution, applied to simulated atomic and molecular lines is presented in Sect. 5. Finally, in Sect. 6, we summarize our conclutions.

\section{Weak line decorrelation and deblending}

We first consider how to extract a proportionality function (Zeeman pattern) that is constant for all lines in a given intensity spectrum. It is obvious that if we assume similar line profiles, i.e. no saturation effects, each line profile is given by the characteristic Zeeman pattern, which we choose to have a central line depth of 1 , "scaled" by the central line depth $d_{\mathrm{c}, i}$ of the current line $i$. For the surrounding local line profile $I_{\text {loc }}$, however, it is more appropriate to consider

$$
\left(1-I_{\mathrm{loc}}(v)\right)=d_{\mathrm{c}, i}\left(1-Z_{I}(v)\right),
$$

rather than $I_{\mathrm{loc}}(v)=d_{\mathrm{c}, i} Z_{I}(v)$. The line depths $d_{\mathrm{c}, i}$ of each line constitute the linemask matrix $M_{I}$ (cf. Sect. 2) with elements $m_{i, I}$

$M_{I}(v)=\sum_{i} m_{I, i} \delta\left(v-v_{i}\right)$,

where $v_{i}$ and $m_{I, i}$ are respectively the position in velocity space and weight of each spectral line. To be able to write Eq. (1) in terms of a linemask, we complete the transformations

$\tilde{I}=1-I \quad$ and $\quad \tilde{Z}_{I}=1-Z_{I}$.

In the weak line approximation, a spectrum can be written in terms of a correlation expression of the so-called line pattern function, or linemask $M$, with the sought-after common line pattern $Z$,

$I=M_{I} * Z_{I}$.

As proposed by Donati et al. (1997), this is equivalent to a (linear) system of equations

\section{$\mathbf{I}=\mathbf{M}_{I} \cdot \mathbf{Z}_{I}$.}

If we know the error $\sigma_{j}$ of each spectral pixel $j$, we can weight each of those equations with the inverse error $1 / \sigma_{j}$ by multiplying both sides with a square diagonal matrix $\mathbf{S}$, where $S_{j j}=$ $1 / \sigma_{j}$. The least squares solution is then given by

$\mathbf{Z}_{I}=\frac{\mathbf{M}_{I}^{T} \cdot \mathbf{S}^{2} \cdot \mathbf{I}}{\mathbf{M}_{I}^{T} \cdot \mathbf{S}^{2} \cdot \mathbf{M}_{I}}$.

If the pixel errors are unknown, $\mathbf{S}$ can be chosen to be the identity matrix and therefore omitted. In our discussion, we do not consider the error matrix, but do remember that if provided, it is important to include it during all further steps.
It should be emphasized here that for intensity spectra, the transformations given by Eq. (3) must be made prior to applying Eq. (5), because for Stokes $I$ the normalized continuum equals 1, instead of zero as for $V, Q$, and $U$.

It is common to interpret Eq. (5) in terms of evaluating the common line pattern only for one single bin $v^{\prime}$ of a given velocity grid at a time, i.e., by transforming local line profiles $I_{\mathrm{loc}, i}$ for each line $i=1, \ldots, n$ into velocity space with their line centers as origin and defining a system of $n$ equations

$$
\begin{gathered}
I_{1}\left(v^{\prime}\right)=m_{1} Z\left(v^{\prime}\right) \\
\vdots \\
I_{n}\left(v^{\prime}\right)=m_{n} Z\left(v^{\prime}\right)
\end{gathered}
$$

In this approach, $\mathbf{M}$ and $\mathbf{I}$ are one-dimensional arrays, and inserting them into Eq. (5) yields the least squares solution of one element of $\mathbf{Z}_{I}$ at a given velocity grid point. Equations (6) assume that there are no blended lines or more precisely that there is only a contribution to $Z$ from one line at a time. The effect of blends is assumed to be averaged statistically. However, in general a measurement at wavelength $\lambda^{\prime}$ corresponding to velocity $v^{\prime}$, theoretically is affected by all lines in the spectrum. An explicit equation for $I$ at $v^{\prime}$ is then

$I\left(v^{\prime}\right)=m_{1} Z\left(v_{1}^{\prime}\right)+m_{2} Z\left(v_{2}^{\prime}\right)+m_{3} Z\left(v_{3}^{\prime}\right)+\ldots$,

where $v_{i}^{\prime}$ is the velocity distance measured from the line center $\lambda_{0, i}$. In practice, most of the contributions are of course negligible. Only terms for which $v_{i}^{\prime}$ lies within the grid of $Z$ are accounted for. Thus, if we assume the first line in our linelist to be blended with the second one, the equation would have to look like

$I\left(v^{\prime}\right)=m_{1} Z\left(v_{1}^{\prime}\right)+m_{2} Z\left(v_{2}^{\prime}\right)$.

The equation for the next pixel at $\lambda^{\prime \prime}\left(\rightarrow v^{\prime \prime}\right)$ is then

$I\left(v^{\prime \prime}\right)=m_{1} Z\left(v_{1}^{\prime \prime}\right)+m_{2} Z\left(v_{2}^{\prime \prime}\right)$.

For some additional pixel at $\tilde{\lambda}$, the contribution from a third line could start to play a role, whereas the first line is already too far away, yielding

$I(\tilde{v})=m_{2} Z\left(\tilde{v}_{2}\right)+m_{3} Z\left(\tilde{v}_{3}\right)$.

This approach not only deconvolves the spectrum for separate lines, but does so it in an analytical way for blended line profiles. The only reason why the previos method yields reasonable results is due to stressed statistics, while here we solve Eq. (5) simultaneously for all components of $Z$. Hence, $\mathbf{M}$ is a matrix of dimensions $p$ times $z$, where $p$ is the number of pixels in the spectrum ( $p$ can be of the order of $10^{5}$ ), and $z$ the number of components for the common line pattern $Z$. However, if two spectral lines are separated by more than two times the velocity limit of $Z$, there will be no equations in the intermediate interval, so the number of equations can be smaller than the number of pixels. The (overdetermined) system of equations then resembles the following (remembering that the indices of $m$ denote the lines in the linemask):

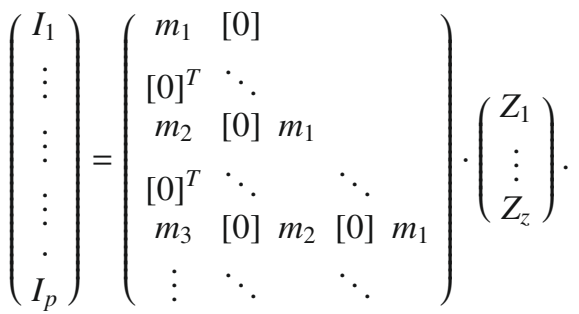




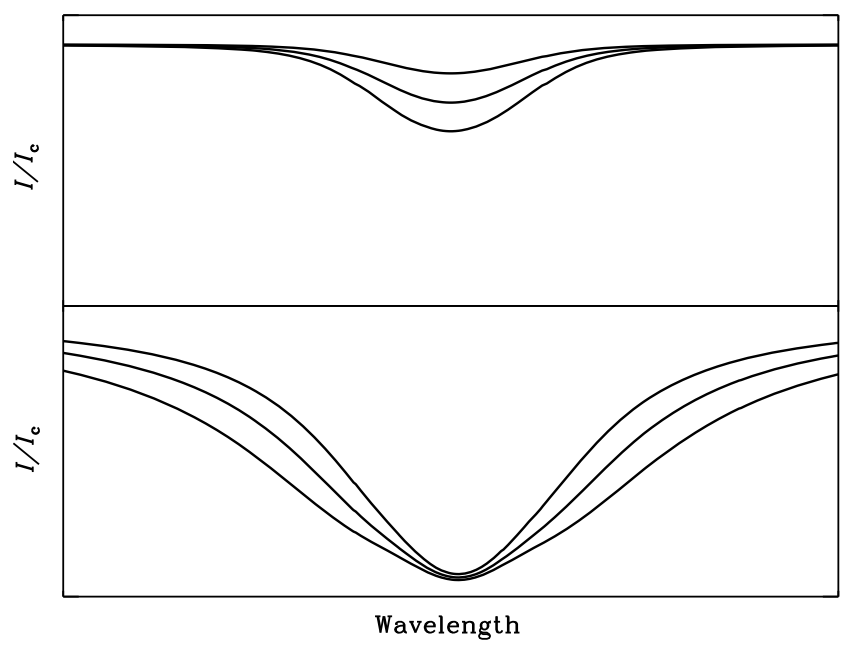

Fig. 1. Changes in the line profile with increasing $\kappa_{\mathrm{l}} / \kappa_{\mathrm{c}}$ in the case of optically thin lines $\left(\kappa_{1} \ll \kappa_{\mathrm{c}}\right.$, upper panel) and optically thick lines $\left(\kappa_{1} / \kappa_{\mathrm{c}} \sim 1\right.$, lower panel $)$.

The diagonal alignment of the weighting factors is clearly evident, since they indicate the pixel at which a specific line begins to contribute to the $Z$-velocity grid, and how it migrates through the bins as we go through our measurement points. We note that the alignment is diagonal strictly only if the distance between two measurement points precisely equals the binsize of our velocity grid, which is not always the case. If the binsize is larger, some weighting factors are allocated one below the other, whereas for too small a binsize, they can be shifted by more than one column.

\section{Optically thin versus optically thick lines}

In Eq. (7), we assume that each datapoint is a linear combination of different contributions from our sought-after common line pattern. According to the Eddingtion-Barbier relation in LTE for the optically thin case, we write for the line depth (Boehm-Vitense 1989)

$R_{\lambda}=\left.\frac{2}{3} \frac{\kappa_{\mathrm{l}}}{\kappa_{\mathrm{c}}} \frac{\mathrm{d} \ln B_{\lambda}}{\mathrm{d} \tau_{\mathrm{c}}}\right|_{\tau_{\mathrm{c}}=2 / 3}$,

where $B_{\lambda}(T)$ is the Planck function, $\kappa_{\mathrm{c}}$ the continuous absorption coefficent, and $\kappa_{1}$ the line absorption coefficient. Equation (12) mainly expresses that for weak lines, the line depth at every point within the line increases in proportion to $\kappa_{\mathrm{l}} / \kappa_{\mathrm{c}}$, or proportionally to the number of atoms $N_{s}$ absorbing in the line. For a blended line profile $R_{\lambda}$ consisting of two lines $R_{1, \lambda}$ and $R_{2, \lambda}$, we may then write

$R_{\lambda}=R_{1, \lambda}+R_{2, \lambda}$.

Thus, it seems obvious that the assumption about linearly added line profiles only holds for weak lines. For optically thick lines, where $\kappa_{1} / \kappa_{\mathrm{c}} \sim 1$ or is greater than 1 , Eq. (12) is no longer a valid approximation, since it can easily produce total line depths $>1$. In general, we search for a line-summing rule for $R_{\mathrm{tot}, \lambda}$ that meets the following criteria:

i) $R_{\mathrm{tot}}\left(R_{1}, R_{2}\right)=R_{\mathrm{tot}}\left(R_{2}, R_{1}\right)$;

ii) $R_{\text {tot }}\left(R_{1}, R_{2}\right)=R_{1}+R_{2}, \quad$ for $\quad R_{1}, R_{2} \ll 1$;

iii) $R_{\text {tot }}\left(1, R_{2}\right)=1, \quad$ for any $R_{2}$;

iv) $R_{\text {tot }}\left(R_{1}, 0\right)=R_{1}$;

v) $R_{\text {tot }}\left(R_{1}, R_{\text {tot }}\left(R_{2}, R_{3}\right)\right)=R_{\text {tot }}\left(R_{\text {tot }}\left(R_{1}, R_{2}\right), R_{3}\right)$.
A simple function exists that satifies the above conditions, i.e., that (i) is symmetric in its arguments; (ii) adds line depths linearly for optically thin lines; (iii) if any of its arguments approaches 1, it does so, too; and according to (iv) and (v) can handle an arbitrary number of lines, even if that number is 1 . This function is as follows:

$R_{\text {tot }}\left(R_{1}, R_{2}\right)=R_{1}+R_{2}-R_{1} \cdot R_{2}$.

Although this formula was derived in a totally heuristic manner, it is obvious that it is able to produce far more reliable results than a simple linear approach, especially when $R_{i} \nless 0.15$ (Unsoeld 1968), because of its second term, which accounts for the effects of line saturation and makes it impossible to reach line depths greater than 1 . Nevertheless, saturation levels in general vary from line to line, and are $<1$. For optically very thick lines for which $\kappa_{\mathrm{l}} / \kappa_{\mathrm{c}}$ at the line center goes to infinity, the residual is approximated by the source function $S_{\lambda}\left(\tau_{\mathrm{c}}=0\right)$, and the limiting central depth is given by $R_{\lambda_{0}}$ in terms of

$R_{\lambda_{0}}=\frac{I_{\mathrm{c}}-S_{\lambda}(0)}{I_{\mathrm{c}}}$

as illustrated in Fig. 1. In the case of NLTE, $S_{\lambda}(0)$ may approach zero, for instance in resonance lines, and $R_{\lambda_{0}}$ becomes 1 , although this is an exceptional case. Attempts to modify Eq. (14) by means of tuning the function towards a more realistic saturation level $<1$ are destined to fail, since any parameter inserted in the second (nonlinear) term would prevent the function from meeting criterium $(\mathrm{v})$.

We instead need another formula that interpolates $R_{\lambda}$ between Eq. (12), which is for both weak lines and the wings of strong lines, and Eq. (15), which is for the optically thick parts of the line. Introducing the quantity

$X(\lambda)=\left.\frac{2}{3} \frac{\kappa_{\mathrm{l}}(\lambda)}{\kappa_{\mathrm{c}}} \frac{\mathrm{d} \ln B_{\lambda}}{\mathrm{d} \tau_{\mathrm{c}}}\right|_{\tau_{\mathrm{c}}=2 / 3}$,

the approximation of the line profile is given by the interpolation formula according to Minnaert (1935):

$R_{\lambda}=\frac{1}{\frac{1}{X(\lambda)}+\frac{1}{R_{\lambda_{0}}}}$.

For strong lines, where $\kappa_{1} / \kappa_{\mathrm{c}}$ is high, $X(\lambda)$ is much larger than 1 , and the term $1 / X(\lambda)$ can be neglected, yielding $R_{\lambda} \approx R_{\lambda_{0}}$. On the other hand, for optically thin lines, $1 / X(\lambda) \gg 1 / R_{\lambda_{0}}$ and $R_{\lambda} \approx X(\lambda)$.

We propose a new application of this formula, which has so far only been employed to calculate equivalent widths and curves of growth. Following our derivation given above, we state that the quantity $X(\lambda)$ can be regarded as the sum of different terms that contribute to the absorption in the line, as an extreme case for instance, the sum over the absorbing atoms. It therefore seems reasonable that the combination of $X_{i}(\lambda)$ for different spectral lines is given by the sum $X_{\text {tot }}(\lambda)=\sum_{i} X_{i}(\lambda)$ of all the contributing quantities. For a blended line profile, we then write

$R_{\lambda, \text { tot }}=\frac{1}{\frac{1}{\sum_{i} X_{i}(\lambda)}+\frac{1}{R_{\lambda_{0}, \text { tot }}}}$.

To find $X_{i}(\lambda)$ as a function of $R_{i, \lambda}$, we simply rearrange Eq. (17) for $X(\lambda)$, yielding

$X_{i}(\lambda)=\frac{1}{\frac{1}{R_{i, \lambda}}-\frac{1}{R_{i, \lambda_{0}}}}$. 
Combining Eqs. (18) and (19) provides a formula that enables us to calculate the line depth at a given wavelength for an arbitrary number of contributing lines, given their individual local line depths and their saturation levels. For a blended line profile, the saturation depth for each contributing line is obtained by interpolating the surfaces as shown in Fig. 2 . The quantity $R_{\lambda_{0}, \text { tot }}$ in Eq. (18) represents the saturation depth of the blended line, and can be calculated similarly by setting $R_{i, \lambda}=R_{i, \lambda_{0}}$, and in turn $R_{i, \lambda_{0}}=1$. By experience, $R_{\lambda_{0} \text {,tot }}$ is always very close to one for a sufficiently large number of blended lines.

To calculate line profiles, we must determine in practice the source function. Only in the case of LTE $S_{\lambda}$ is known (namely $S_{\lambda}=B_{\lambda}$ ). For the Milne-Eddington Model of line transfer, Mihalas (1978) provides a formula for the saturation depth of a line that depends only on temperature and wavelength, and neglects scattering in both the continuum and line (assuming LTE in the line). In our case, we often deal with strong lines that do not form in LTE and scattering is present. This approach then seems unsatisfactory also when we take into account that level population depends on the excitation energy of the ground state for a given electronic transition. Therefore, to estimate saturation depths as accurately as possible, we employed the code STOPRO (Solanki 1987; Frutiger et al. 2000; Berdyugina et al. 2003), solving the polarized radiative transfer equations for 33 different elements and ions, 6 stellar atmospheric models (Kurucz 1993), 31 wavelengths covering the region from 4000 to $10000 \AA$, and every possible lower excitation energy starting from $0.1 \mathrm{eV}$ and increasing in $0.3 \mathrm{eV}$ steps (the maximal lower excitation energy for an ion is of course lower than the next ionization energy level).

To combine all formulae together, we recall our empirical line-summing formula in Eq. (14), where we had to assume that all saturation depths equal one, and compare this with the interpolation formulae Eqs. (18) and (19). We expand Eq. (18) into a Taylor series for two lines $\left[R_{1}(\lambda), R_{2}(\lambda)\right]:=\boldsymbol{R}$, by setting $R_{1, \lambda_{0}}$ and $R_{2, \lambda_{0}}$ equal to 1 and obtain

$$
\begin{aligned}
R_{\mathrm{tot}} & =\frac{1}{\frac{1}{\frac{1}{1 / R_{1}-1}+\frac{1}{1 / R_{2}-1}}+1}=\frac{R_{1}+R_{2}-2 \cdot R_{1} \cdot R_{2}}{1-R_{1} \cdot R_{2}} \\
& =\sum_{|\alpha|} \frac{\left.\mathrm{D}^{\alpha} R_{\mathrm{tot}}(\boldsymbol{R})\right|_{[\boldsymbol{R}=0]}}{\alpha !} \cdot \boldsymbol{R}^{\alpha}=R_{1}+R_{2}-R_{1} \cdot R_{2}+\ldots
\end{aligned}
$$

We can immediately see that Eq. (14) represents the first two non-zero terms of the Taylor expansion of the interpolation formula. Our estimation was therefore quite accurate, and can be helpful in practice, if saturation levels are unknown, and one benefits from the simplicity of Eq. (14).

\section{Numerical implementation}

A common problem in spectral analysis is optimal binning. Spectral lines in the blue part of a spectrum are narrower than those at red wavelengths, since (non-transitional) broadening effects depend on wavelength. After transforming the wavelength scale into the velocity domain centered on the line wavelength, we can compare line profiles from different wavelength regions. However, any predefined velocity grid (usually at equidistant points) will never match measured data points transformed into the velocity space of a given line. The easiest way is to simply assign the measured velocity to the closest gridpoint. In such a case, however, one disregards the very accurate wavelength information of a spectrum (accurate to a fraction of an Angstrom).

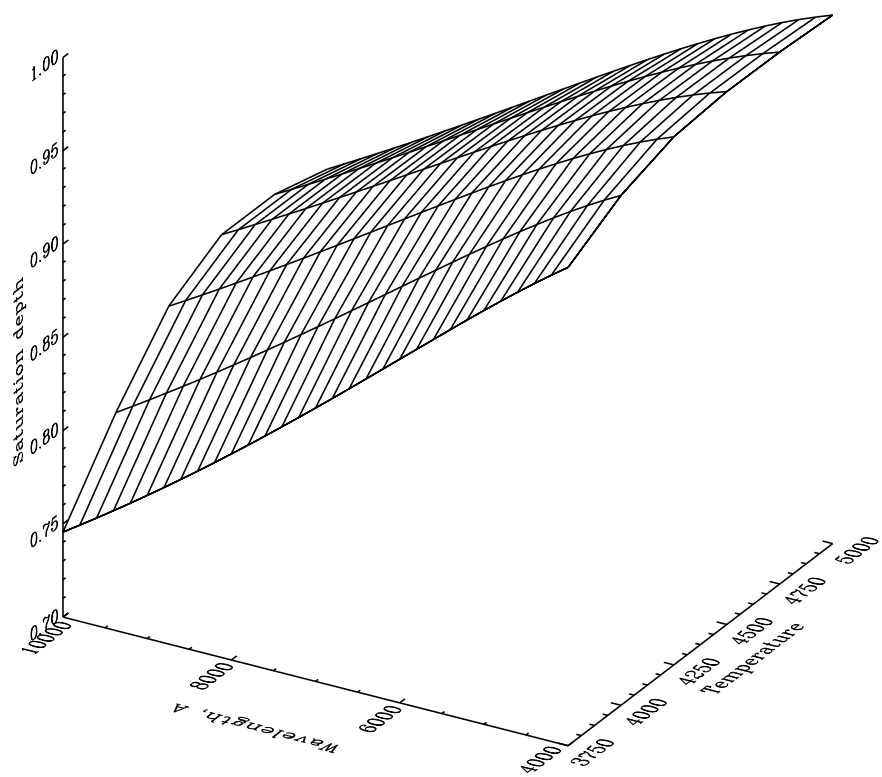

Fig. 2. Saturation levels for Fe I lines with lower excitation energies of $4.6 \mathrm{eV}$ calculated by the code STOPRO.

Here we propose to employ inverse interpolation, allowing multiple gridpoints to incorporate information of one datapoint.

We implemented quadratic inverse interpolation for NDD and the methods used for comparison, yielding a noticeable improvement in retrieved common line patterns. A detailed description is given in Appendix A, where we also present a practical algorithm for inverse spline interpolation.

Since Eqs. (14) and (18) are nonlinear functions, there is no matrix representation that would enable us to employ Eq. (5) to find a solution in $Z$. Thus, we have to apply iterative methods to approach a least squares solution in $Z$. We intentionally do not write the solution, because we show later that, depending on how densely lines are blended with each other, multiple minima may appear in the topology of solutions. In Appendix B, we present two algorithms implemented to find a minimum; the Gauss-Newton with Minimization (GNwM) method and the Levenberg-Marquardt (LM) method. Although the latter is in general preferable because it minimizes the $\chi^{2}$ merit function far more efficiently, the former should not be underestimated because of its simplicity.

Another problem, which is atypical in the optimization of functions, is that we do not have a "model" function that depends on our $z$ parameters, but rather it is the true form of our function depending on the number of parameters that is unknown. Therefore, the derivatives necessary for the Jacobi and Hessian matrices in the solving algorithms are also unknown, and need to be determined for each equation separately. Iterative evaluation of derivatives would cause our code to be extremely slow since we consider $10^{4}$ equations and more, depending on as many as several hundred parameters. Therefore, we resolved this problem analytically, by determining all partial derivatives when we defined our system of nonlinear equations, depending on the number of contributing blends and the different gridpoints that they contribute to (see Appendix C). We note that these two numbers are not necessarily equal, which is the case for only two line centers lying within the velocity bin, and causes additional problems in finding the derivatives. 


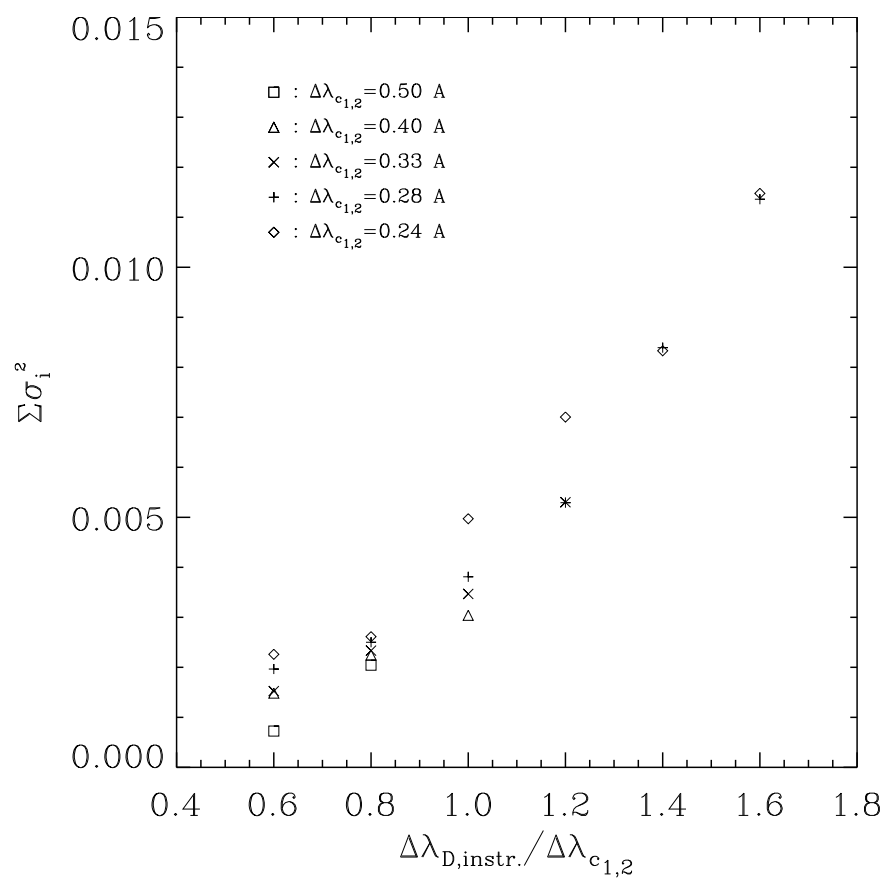

Fig. 3. Performance of our NDD method in recovering common line patterns from a blended line profile with line center separation $\Delta \lambda_{c_{1,2}}$ and effective broadening $\Delta \lambda_{D \text {,instr. }}$.

\subsection{Critical blends}

As we have mentioned, the ability of a deconvolution method to identify a common line pattern in blended line profiles depends on the proximity of the line centers. For instance, if we have dozens of lines that are broadened significantly and separated by only fractions of an Angstrom, they would form a broad "valley" with many degenerate solutions for the instrinsic line profile. We approximate the Doppler broadening of a line by

$\Delta \lambda_{\mathrm{D}}=\frac{\lambda}{c} \cdot \sqrt{\xi_{\mathrm{th}}^{2}+\xi_{\mathrm{turb}}^{2}+(v \sin i)^{2}}$,

where $\xi_{\text {th }}=\sqrt{2 R T / \mu}$ is the mean thermal velocity of an atom, $\xi_{\text {turb }}$ accounts for the turbulent motions in the stellar atmosphere, and $v \sin i$ represents the stellar rotation. To allow for instrumental broadening, we adopt

$\left(\Delta \lambda_{\mathrm{D}, \text { instr }}\right)^{2}=\left(\Delta \lambda_{\mathrm{D}}\right)^{2}+\left(\Delta \lambda_{\text {instr }}\right)^{2}$.

For a separation of $\Delta \lambda_{\mathrm{c}_{1,2}}$ between the line centers of two spectral lines, influenced by the above broadening effects, we claim that the performance of a deconvolution code theoretically depends on the ratio

$Q_{1,2}=\Delta \lambda_{\text {D,instr }} / \Delta \lambda_{c_{1,2}}$.

Figure 3 illustrates the ability of our deconvolving code to recover the original line profiles. We blend two identical line patterns, apply our code and compare the retrieved profile to the original single input pattern. We plot the sum of the variance $\left(\sum \sigma_{i}^{2}\right)$ as a function of total line broadening in units of line separation (Eq. (23)). For a given $Q_{1,2}$, the ability to recover the original single line pattern is roughly constant, but errors then begin to increase rapidly if $Q_{1,2} \geq 1$. We emphasize that this limit does not depend on the method, but that the absolute value of the error does! In practice, however, results depend on the distance of line centers. For a given ratio $Q_{1,2}$ and spectral resolution $R$, fewer pixels carry information about the line, if $\Delta \lambda_{c_{1,2}}$

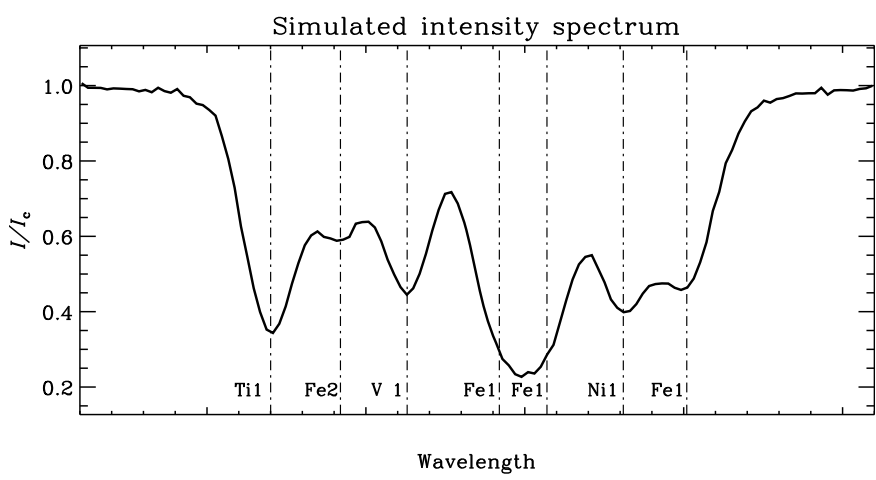

Fig. 4. Simulated intensity spectrum of 7 blended lines. The dashed vertical lines mark the line centers; element names and ionization levels are labelled at the bottom.

is small compared to the situation where $\Delta \lambda_{c_{1,2}}$ is large. That is, the (intensity) gradient in the pixel frame is far steeper, imposing difficulties for the applied LSD method in recovering details from the common line profile and resulting in larger errors per pixel.

\section{Results}

\subsection{Atomic profiles: Stokes I}

We simulated an intensity spectrum containing 7 blended spectral lines (Fig. 4), among them two iron ions, as well as vanadium and nickel, each with very different lower excitation energies (to test the reliability of the Minnaert approach and its dependence on individual saturation levels, see Eq. (18)), to probe the functionality of both the different approaches and the nonlinear solving methods. This does not represent a true spectrum since the real line centers were shifted, to produce a challenging blended profile. Therefore, the abscissa marks are omitted, both $v \sin i$ and instrumental broadening were assumed to have particular values, and we assumed a noise level of $\sim 0.5 \%$. The line central depths $d_{\mathrm{c}, i}$ range from 0.38 to 0.66 , and they were "measured" by simulating single line profiles. We emphasize that the deconcolving performance relies on precise knowledge of the parameters defining the weighting factors $m_{i}$. For Stokes $I$, the line central depth is the only determining parameter, and, as can be seen in Eq. (1), if they are concordant with the (well-normalized!) spectrum, the recovered Zeeman pattern should have normalized wings and a central line depth $d_{c, Z}$ exactly equal to 1 , regardless of the strength of the original line depths. Only such a common line pattern, scaled to individual line depths, and treated according to Eqs. (18) and (19) for blended profiles, will be able to reproduce the original spectrum. Therefore, the true central line depth of the retrieved Zeeman pattern is a strong indication of the effectiveness of a multi-line method.

We applied the conventional LSD ("normal LSD"), given by Eq. (6), and the deblending, yet linear method, corresponding to Eq. (11). Both methods can be solved using Eq. (5). We compare them to our NDD method according to our realization of the Minnaert interpolation formula, applying both the Gauss-Newton with Minimization and the Levenberg-Marquardt method for the iterative solving of the nonlinear sets of equations (see Fig. 5).

"Normal LSD" (dash-dotted curve at the bottom), which is not a true deconvolution method, fails in this attempt, when all lines are heavily blended, resulting in strong wiggles in the wings, a continuum level dependent on the number of blended 


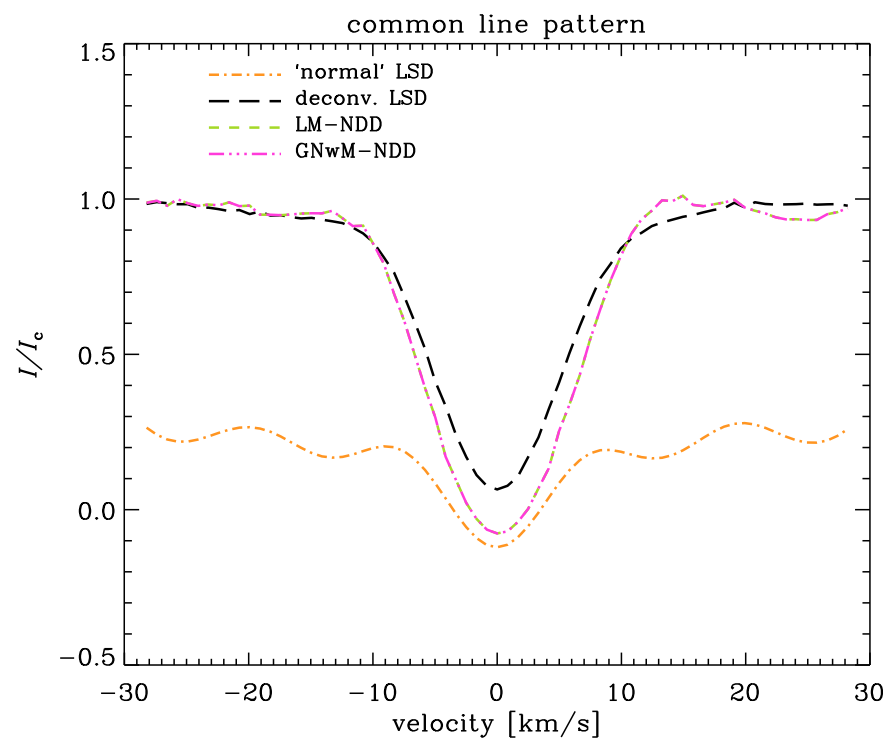

Fig. 5. Recovered intensity pattern from different methods employed to the atomic spectrum given in Fig. 4.

lines, if there is a noticeable continuum at all, and a central line depth significantly smaller than 1 . The other linear, but deconvolution approach (dashed line) is far more successfull, but the wings are (asymmetrically) broadened, and the line center does not reach 0 . Both of these results infer that the linear approach for this spectrum is unsuccessfull, because the absorbing region is entirely optically thick. When the Minnaert formula and inverse quadratic interpolation are applied, as presented in Sect. 4, the result is a noticeably smoother profile compared to a "nearest bin" attempt. The nonlinear (NDD) method reproduces a fairly perfect profile within the line, showing a central line depth $\simeq 1$. The differences between the two solving numerical methods are marginal, so the two profiles almost coincide. However, in the far wings of the Zeeman pattern, which are arguably already in the continuum, the nonlinear solving causes errors, because of the handling of small numbers (if we recall that we operate with $1-I)$. This problem should be solved by applying boundary conditions to the continuum part of the profile.

\subsection{Molecular profiles: Stokes I}

In molecular bands and especially in the band head, a large number of blends contribute to the measured line profile, and serious deconvolution and provision for nonlinearity become mandatory. To illustrate the capabilities of our new method, we applied the method to a simulated molecular $\mathrm{TiO} \gamma(0,1)$ band at $7591 \AA$, as shown in Fig. 6. Our NDD technique recovers a common line pattern that almost coincides with the input Z-profile except for the right wing of the line. This is due to the band head, where the bulk of blends interfere with each other in a way that basically removes the individual profiles, precluding a unique solution. As mentioned before, this problem increases for greater broadening and more significant blending. Nevertheless, our method is capable of retrieving the necessary details of the line profile (e.g., width, see Fig. 7), which features a line central depth of virtually equal 1 , and being easily able to identify possible irregularities within the entire profile. A comparison with the results for the linear attempt shows that the effects of nonlinearity become noticeable in terms of their conspicuously asymmetric wings and

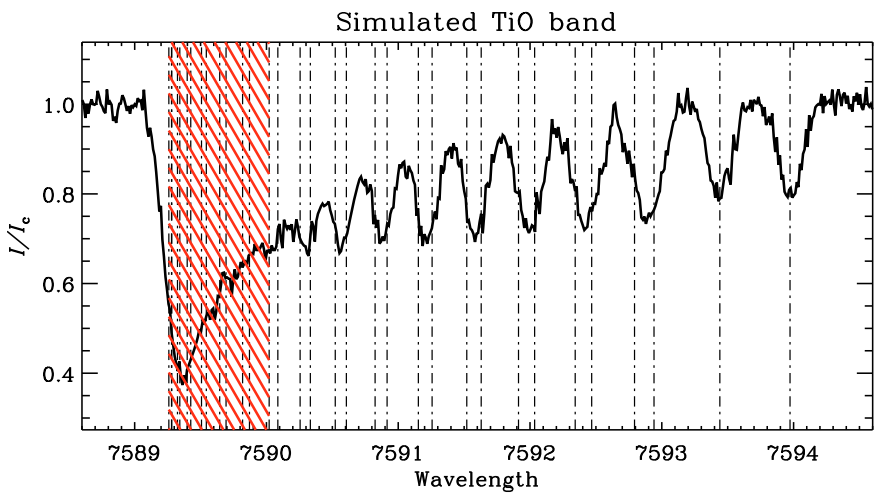

Fig. 6. Simulated molecular $\mathrm{TiO} \gamma(0,1)$ band, including Doppler broadening and $\sim 1 \%$ noise level. The dashed-dotted vertical lines indicate the line centers. The hashed region of the band head is where many blends are packed together and $Q_{i, j} \gg 1$.

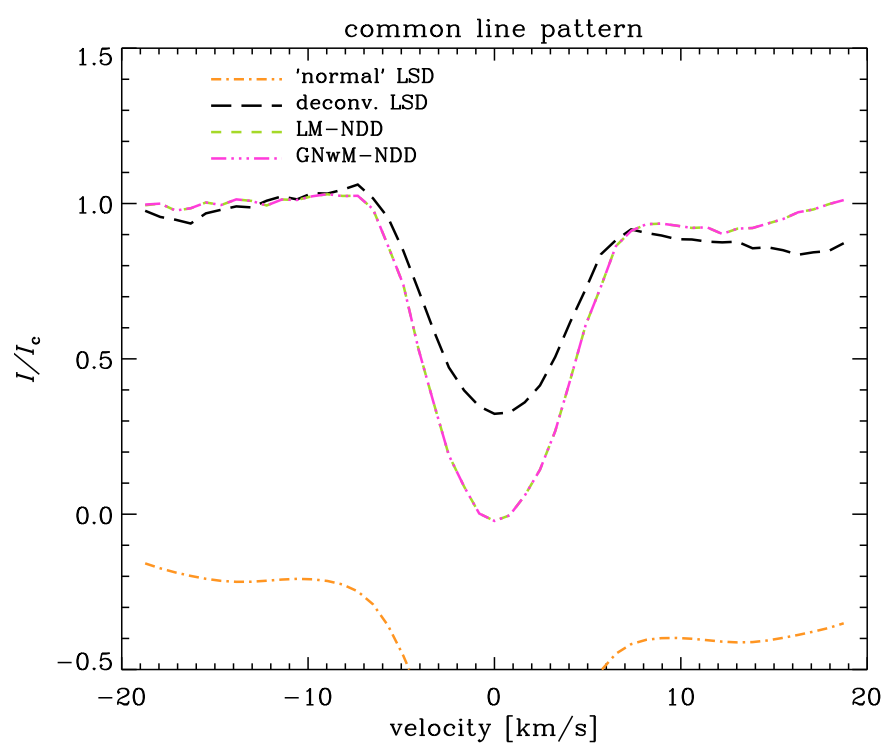

Fig. 7. Recovered intensity pattern from different methods employed to study the molecular band given in Fig. 6.

shallow central profile. Again, "normal LSD" fails, because it lacks deconvolving ability.

\section{Conclusion}

Our nonlinear approach to deconvolving blended line profiles by applying the Minneart interpolation formula, combined with the advantages provided by inverse interpolation, represents a significant revision of least squares deconvolution techniques, mainly because the latter do not incorporate a deconvolution of blended lines. Given a linemask that includes all relevant atomic or molecular data, we are able to disentangle arbitrarily heavily blended spectra in a physically consistent manner, fully abandoning any preliminary assumptions about the intrinsic single line profile, apart from its existence. This allows us to retrieve the true common line patterns even from a limited number of blended lines and will enable (Zeeman) Doppler Imaging to be applied even to noisy datasets of narrow spectral intervals.

Our method is also able to properly disentangle molecular bands consisting of many tens of blended line profiles within a narrow spectral range. Heavily broadened molecular spectra do not offer, however, a unique solution for the recovery of an intrinsic line profile: results always show depressed right wings. 
For the time being, it is nonetheless the tool most capable of identifying weak features in molecular absorption lines.

We place emphasis on the importance of a proper deconvolution of the spectrum to be analyzed, highlighting the need to disentangle blends. As shown in the derived common line patterns in Sect. 5, any non-deconvolving method imposes additional line broadening by neglecting the influence of close-by blends. In a forthcoming paper, we discuss whether ZDI codes based on these methods infer stellar spots at systematically higher lattitudes, due to a technical artifact.

The method described in the present paper offers a general and direct formalism concerning how spectral line parameters, as well as ambient and instrumental effects affect an observed intensity profile. It can be used to address a variety of problems in stellar spectrum analysis, and it is likely that it will find more general applications in many types of spectroscopy, where the quality of data is insufficient to apply direct fitting of spectra.

Acknowledgements. This work is supported by the EURYI (European Young Investigator) Award provided by the European Science Foundation (see www.esf.org/euryi http://www.esf.org/euryi) and SNF grant PE002-104552.

\section{Appendix A: Inverse interpolation}

Each datapoint in a spectrum has a distinct distance in wavelength to the center of line $\lambda_{0, i}$ for the spectral line $i$, which in turn corresponds to a distance in velocity space $\Delta v_{i}$. The grid for which we wish to retrieve our common line pattern $Z$ consists of equidistant points, in other words, bins of constant binsize $\Delta_{\text {bin }}$. If we consider $\Delta_{\text {bin }} \equiv 3 \mathrm{~km} \mathrm{~s}^{-1}$ and a pixel with $\Delta v_{i}=5.1 \mathrm{~km} \mathrm{~s}^{-1}$, one is willing to argue that it corresonds to the gridpoint $Z\left(v=6 \mathrm{~km} \mathrm{~s}^{-1}\right)$, simply because it is the closest one. However, one could instead inversely interpolate, so as to express the datapoint in terms of multiple $Z$-gridpoints. Applying quadratic interpolation, we obtain

$Z(v=5.1)=p_{0} \cdot Z(v=3)+p_{1} \cdot Z(v=6)+p_{2} \cdot Z(v=9)$,

where the constants $p_{0}, p_{1}, p_{2}$ can be calculated with the Lagrange-formula for interpolation:

$p_{k}(v)=\frac{\left(v-v_{0}\right) \cdot \ldots \cdot\left(v-v_{k-1}\right)\left(v-v_{k+1}\right) \cdot \ldots \cdot\left(v-v_{n}\right)}{\left(v_{k}-v_{0}\right) \cdot \ldots \cdot\left(v_{k}-v_{k-1}\right)\left(v_{k}-v_{k+1}\right) \cdot \ldots \cdot\left(v_{k}-v_{n}\right)}$,

yielding

$Z(v=5.1)=0.195 Z(v=3)+0.91 Z(v=6)-0.105 Z(v=9)$

Another method that was implemented was that of spline interpolation, since we expect our Zeeman pattern to be smooth and continuous. The difficulty lies in analytically solving the linear system of equations with its typical tridiagonal, strictly diagonally dominant $(z-2) \times(z-2)$ matrix (again, assuming that $\Delta_{\text {bin }}=$ constant $)$ :

$p_{i-1}+4 p_{i}+p_{i+1}=\frac{6}{\left(\Delta_{\text {bin }}\right)^{2}}\left(Z_{i+1}+Z_{i-1}\right)=A_{i}, \quad i=1, \ldots, n-1$,

and $p_{0}=p_{n}=0$, for $n+1=z$ knots (gridpoints). The cubic polynomials defining the spline are known to be given as

$$
\begin{aligned}
S_{i}(v)= & \frac{p_{i+1}\left(v-v_{i}\right)^{3}+p_{i}\left(v_{i+1}-v\right)^{3}}{6 \Delta_{\text {bin }}}+\left(\frac{Z_{i+1}}{\Delta_{\text {bin }}}-\frac{\Delta_{\text {bin }}}{6} p_{i+1}\right)\left(v-v_{i}\right) \\
& +\left(\frac{Z_{i}}{\Delta_{\text {bin }}}-\frac{\Delta_{\text {bin }}}{6} p_{i}\right)\left(v_{i+1}-v\right) .
\end{aligned}
$$

After Gauss elimination, Eq. (A.4) can be written as

$$
\left(\begin{array}{cccc}
4-\frac{0}{1} & 1 & 0 & \\
0 & 4-\frac{1}{4} & 1 & \ddots \\
& 0 & 4-\frac{4}{15} & \ddots \\
& & \ddots & \ddots
\end{array}\right)\left(\begin{array}{c}
p_{1} \\
\vdots \\
p_{n-1}
\end{array}\right)=\left(\begin{array}{c}
A_{1} \\
A_{2}-\frac{1}{4} A_{1} \\
A_{3}-\frac{4}{15}\left(A_{2}-\frac{1}{4} A_{1}\right) \\
A_{4}-\frac{15}{56}\left(A_{3}-\frac{4}{15}\left(A_{2}-\frac{1}{4} A_{1}\right)\right) \\
\vdots
\end{array}\right)
$$

To express the coefficients $p_{i}$ after back-substitution of this linear system of equations, we introduce the recursive sequences

$$
\begin{aligned}
& q_{i+1}=4 q_{i}-q_{i-1}, \quad q_{0}=0, q_{1}=1 \\
& r_{i+1}=A_{i+1}-\frac{q_{i}}{q_{i+1}} r_{i}, \quad r_{1}=A_{1} .
\end{aligned}
$$

Using this, we can write for $p_{i}, i=2, \ldots, n$ :

$p_{i-1}=\frac{r_{i-1}-p_{i}}{4-\frac{q_{i-2}}{q_{i-1}}}$

or, without the use of the $r_{i}$ 's:

$p_{i-1}=\frac{-q_{i-1} p_{i}+\sum_{k=1}^{i-1}(-1)^{i-1-k} q_{k} A_{k}}{q_{i-1}}$.

We have presented the sought-after coefficients in Eq. (A.5) in terms of the quantities $A_{i}$, where the $A_{i}$ 's in turn are linearly dependent on $Z_{i}$, as given in Eq. (A.4). Even if a datapoint is affected by only one spectral line, it will then be represented as a linear combination of all elements of $Z$, where in the case of quadratic interpolation, it will always be represented by three of them.

\section{Appendix B: Solving algorithms}

For the Gauss-Newton method, following Nipp (2002), we represent our set of equations by

$\frac{f_{i}(\mathbf{Z})-y_{i}}{\sigma_{i}}=r_{i}$

where $y_{i}$ are the measurement values and $r_{i}$ the residuals. We search for a $\mathbf{Z} \in \mathbb{R}^{z}$ for which $\|\mathbf{r}\|_{2}^{2}=\chi^{2}(\mathbf{Z})$ is minimal. We complete a Taylor expansion around a starting value $\mathbf{Z}_{\text {cur }}$ (second and higher order terms (h.o.t.) are neglected) to obtain

$\mathbf{f}(\mathbf{Z})=\mathbf{f}\left(\mathbf{Z}_{\text {cur }}+\boldsymbol{\xi}\right)=\mathbf{f}_{\text {cur }}+\mathbf{A}_{\text {cur }} \boldsymbol{\xi}+$ h.o.t.,

where $\mathbf{A}_{\text {cur }} \in \mathbb{R}^{p \times z}$ denotes the Jacobi matrix at $\mathbf{Z}_{\text {cur }}$, and inserting it into Eq. (B.1) yields the linearized residual equations

$\mathbf{S} \cdot\left(\mathbf{A}_{\mathrm{cur}} \boldsymbol{\xi}+\mathbf{f}_{\mathrm{cur}}-\mathbf{y}\right)=\boldsymbol{\rho}_{\mathrm{cur}}$

where $S_{j j}=1 / \sigma_{j}$ is the diagonal matrix containing the inverse pixel errors. This set of linear equations can be solved by applying Eq. (5). We then derive $\boldsymbol{\xi}_{\text {next }}$ and thus an improved approximation $\mathbf{Z}_{\text {next }}=\mathbf{Z}_{\text {cur }}+t \boldsymbol{\xi}_{\text {next }}$, where we define the parameter $t$ so that $t \boldsymbol{\xi}_{\text {next }}$ represents the direction of the steepest descent. That is, for $t=1, \frac{1}{2}, \frac{1}{4}, \ldots$ we test whether

$\chi^{2}\left(\mathbf{Z}_{\text {cur }}+t \boldsymbol{\xi}_{\text {next }}\right)<\chi^{2}\left(\mathbf{Z}_{\text {cur }}\right)$.

After $k$ iterations, we then force $\mathbf{Z}_{k}$ to converge and assume that $\boldsymbol{\rho}_{k} \simeq \mathbf{r}_{k}$. The disadvantage of the steepest descent methods is that the new gradient at the minimum point of any line minimization is perpendicular to the direction just traversed. If not, the new gradient would have a nonzero component along the 
previous direction, which we claimed to have traversed until the minimum, and so the new direction would not be the steepest gradient. Therefore, one must make a right angle turn, which does not, in general, lead to the minimum.

The Levenberg-Marquardt method is more efficient, because of its favorable direction of descent. Again, we define the $\chi^{2}$ merit function in terms of least squares to be

$\chi^{2}(\mathbf{Z})=\sum_{i=1}^{p}\left[\frac{y_{i}-f_{i}(\mathbf{Z})}{\sigma_{i}}\right]^{2}$.

Sufficiently close to the minimum, we expect the $\chi^{2}$ function to be well approximated by a quadratic form, according to Press (1992)

$\chi^{2}(\mathbf{Z}) \approx \gamma-\boldsymbol{d} \cdot \mathbf{Z}+\frac{1}{2} \mathbf{Z} \cdot \mathbf{D} \cdot \mathbf{Z}$,

where $\boldsymbol{d}$ is an $z$-vector and $\mathbf{D}$ is an $z \times z$ matrix. If Eq. (B.6) is a good approximation, then one leap will take us from the current values $Z_{\text {cur }}$ to the minimizing ones $Z_{\min }$, i.e.,

$\mathbf{Z}_{\min }=\mathbf{Z}_{\mathrm{cur}}+\mathbf{D}^{-1} \cdot\left[-\nabla \chi^{2}\left(\mathbf{Z}_{\mathrm{cur}}\right)\right]$

However, if (B.6) does not represent the shape of $\chi^{2}$ at $Z_{\text {cur }}$, i.e., when we are far from the minimum, then all we can do is apply the steepest descent method, as we did for the Gauss-Newton minimization

$\mathbf{Z}_{\text {next }}=\mathbf{Z}_{\text {cur }}-$ constant $\cdot \nabla \chi^{2}\left(\mathbf{Z}_{\text {cur }}\right)$.

The Levenberg-Marquardt method adopts both extremes, by varying smoothly between the inverse-Hessian method (B.7) and the steepest descent method (B.8), depending on how far we are from the minimum. In addition, it provides an estimated covariance matrix of the standard errors in the fitted values $Z$.

\section{Appendix C: Derivative of the extended interpolation formula}

For our solving algorithms, we develop the partial derivatives for each equation with respect to each element of our sought-after common line pattern $Z(v)$ to produce the Jacobi matrix and the Hessian matrix, which can also be approximated by the first derivatives. If we define $d_{j}=1 / R_{j, \lambda_{0}}$ for $1 \leq j \leq z$, where $z$ is the number of parameters in $Z$, and $d_{\text {tot }}=1 / R_{\lambda_{0} \text {,tot }}$, then we rewrite Eq. (19) with the weights $m_{j}$ as

$X_{j}=\frac{m_{j} Z_{j}}{1-d_{j} m_{j} Z_{j}}$

and Eq. (18), for $1 \leq i \leq p$, where $p$ equals the number of equations, as

$f_{i}=\frac{s_{i}}{1+d_{\mathrm{tot}} s_{i}}, \quad$ with $\quad s_{i}=\sum_{j \in[j]_{i}} X_{j}$

where $[j]_{i}$ is the list of line-indices that contribute to the $i$ th equation. The partial derivatives are then given by

$\frac{\partial f_{i}}{\partial s_{i}}=\frac{\left(1+d_{\mathrm{tot}} s_{i}\right)-d_{\mathrm{tot}} s_{i}}{\left(1+d_{\mathrm{tot}} s_{i}\right)^{2}}=\frac{1}{\left(1+d_{\mathrm{tot}} s_{i}\right)^{2}}$
$\frac{\partial s_{i}}{\partial Z_{j}}=\frac{m_{j}\left(1-d_{j} m_{j} Z_{j}\right)+m_{j} d_{j} m_{j} Z_{j}}{\left(1-d_{j} m_{j} Z_{j}\right)^{2}}=\frac{m_{j}}{\left(1-d_{j} m_{j} Z_{j}\right)^{2}}$

Therefore, for the elements of the Jacobi matrix $(\partial f / \partial Z)_{i j}$ we find that

$\frac{\partial f_{i}}{\partial Z_{j}}=\frac{\partial f_{i}}{\partial s_{i}} \frac{\partial s_{i}}{\partial Z_{j}}=\frac{m_{j}}{\left(1+d_{\mathrm{tot}} s_{i}\right)^{2}\left(1-d_{j} m_{j} Z_{j}\right)^{2}}$.

\section{References}

Berdyugina, S. V., Solanki, S. K., \& Frutiger, C. 2003, A\&A, 412, 513

Boehm-Vitense, E. 1989, Introduction to stellar astrophysics (New York: Cambridge University Press)

Donati, J. F., Semel, M., Carter, B. D., Rees, D. E., \& Cameron, A. C. 1997, 291, 658

Frutiger, C., Solanki, S. K., Fligge, M., \& Bruls, J. H. M. J. 2000, A\&A, 358, 1109

Kurucz, R. L. 1993, CDROM No. 13

Martínez Gonzáles, M. J., Asensio Ramos, A., Carroll, T. A., et al. 2008, 486, 637

Mihalas, D. 1978, Stellar atmospheres (San Francisco: W. H. Freeman and Company)

Minnaert, M. 1935, 10, 40

Nipp, K. 2002, Lineare Algebra (Zurich: vdf)

Press, W. H. 1992, Numerical Recipes (New York: Cambridge University Press) Semel, M. 1989, 225, 456

Semel, M., \& Li, J. 1996, Sol. Phys., 164, 417

Solanki, S. K. 1987, Ph.D. Thesis, ETH, Zurich, Switzerland

Unsoeld, A. 1968, Physik der Sternatmosphaeren (Berlin: Springer-Verlag) 